\title{
Adrenal Venous Sampling in Patients with Primary Aldosteronism: Which Is the Best Evaluation Method for Laterality Assessments?
}

\author{
Kimei Azama', Masahiro Okada ${ }^{2}$, Akira Yogi ${ }^{1}$, Tomomi Koga ${ }^{3}$, Yuko Iraha ${ }^{1}$, Joichi Heianna ${ }^{1}$, \\ Hideaki Nakamura ${ }^{4}$, Hiroaki Masuzaki ${ }^{4}$, Sadayuki Murayama ${ }^{1}$ \\ ${ }^{1}$ Department of Radiology, Graduate School of Medicine, University of the Ryukyus, Nishihara, Japan \\ ${ }^{2}$ Department of Radiology, Nihon University School of Medicine, Tokyo, Japan \\ ${ }^{3}$ Department of Radiology, Nakagami Hospital, Okinawa, Japan \\ ${ }^{4}$ Division of Endocrinology, Diabetes and Metabolism, Hematology and Rheumatology (Second Department of Internal Medicine), \\ Graduate School of Medicine, University of the Ryukyus, Nishihara, Japan \\ Email: okinawan@iemik.onmicrosoft.com, *okada.masahiro@nihon-u.ac.jp, ayogi@med.u-ryukyu.ac.jp, \\ tomozo_kg1030@yahoo.co.jp, irayu@med.u-ryukyu.ac.jp, jana@mx51.et.tiki.ne.jp, ryudai2ndint_2008_1979@yahoo.co.jp, \\ hiroaki@med.u-ryukyu.ac.jp, sadayuki@med.u-ryukyu.ac.jp
}

How to cite this paper: Azama, K., Okada, M., Yogi, A., Koga, T., Iraha, Y., Heianna, J., Nakamura, H., Masuzaki, H. and $\mathrm{Mu}-$ rayama, S. (2017) Adrenal Venous Sampling in Patients with Primary Aldosteronism: Which Is the Best Evaluation Method for Laterality Assessments? Open Journal of Radiology, 7, 219-227.

https://doi.org/10.4236/ojrad.2017.74024

Received: September 6, 2017

Accepted: November 28, 2017

Published: December 1, 2017

Copyright $\odot 2017$ by authors and Scientific Research Publishing Inc. This work is licensed under the Creative Commons Attribution International License (CC BY 4.0).

http://creativecommons.org/licenses/by/4.0/

\section{(c) (i) Open Access}

\begin{abstract}
Purpose: The purpose was to investigate the most accurate method of adrenal venous sampling to diagnose unilateral primary aldosteronism (PA) prior to laterality assessment. Materials and Methods: Sixty-four consecutive PA patients were enrolled in this retrospective study. A catheter was placed in the common-trunk-vein (CTV), below the confluence of the inferior-phrenic-vein and the central-adrenal-vein (CAV) and the right-adrenal-vein (RAV). Bloodsampling was performed both pre- and post-adrenocorticotropic hormone stimulation. Lateralized ratio [LR; aldosterone/cortisol ratio (ACR) on highvalue side/ACR on low-value side], contralateral ratio (CR; ACR on low-value side/ACR on inferior-vena-cava ratio), and plasma aldosterone concentration (PAC) were evaluated. The diagnostic accuracy of LR, CR and PAC in CTV/ $\mathrm{CAV} / \mathrm{RAV}$ during pre- and post-ACTH was compared by receiver-operatingcharacteristic (ROC) analysis. Results: LR-CAV post-ACTH showed the highest detection rate for unilateral adrenal lesions (93.3\%; 14/15), with a sensitivity of 0.93 and a specificity of 0.84 at a cut-off value of 2.5. CR-post-ACTH had the highest $A z$ value (0.89), with a detection rate of $86.7 \%(13 / 14)$, a sensitivity of 0.98 , and a specificity of 0.88 at a cut-off value of 0.8 . Conclusion: CR-post-ACTH and LR-CAV-post-ACTH are accurate predictors for laterality assessment in PA.
\end{abstract}




\section{Keywords}

Adrenal Venous Sampling, Primary Aldosteronism, Hypertension, Adrenal Grand

\section{Introduction}

Primary aldosteronism (PA) is defined as "the group of disorders in which aldosterone production is inappropriately high, relatively autonomous from the renin-angiotensin system, and non-suppressible by sodium loading" by the American Endocrine Society Guidelines [1]. Several recent studies have suggested that the prevalence of PA is higher $(3.2 \%-21.7 \%)$ in patients with secondary hypertension than previously reported [2]. It is important to diagnosis PA from essential hypertension since PA patients have a higher cardiovascular complication rate than patients with essential hypertension [3]. Both of aldosteroneproducing adenomas (APA) and bilateral idiopathic hyperaldosteronism (IHA) are the most common subtypes of PA.

The management of PA depends on whether adrenal gland is involved unilaterally or bilaterally. PA patients with unilateral involvement are managed surgically (e.g. laparoscopic adrenalectomy), whereas those with bilateral involvement are medically managed (e.g. with mineralocorticoid receptor antagonists). Hypertension usually improves dramatically after surgery in most of the patients with unilateral APA [4]. So, accurate laterality assessments contribute to the appropriate treatment, which improve the prognosis of PA patients.

However, it is often difficult to evaluate the laterality by conventional imaging modalities. It has been reported that microadenomas less than $6 \mathrm{~mm}$, which account for nearly $50 \%$ of APA [5], are difficult to detect on CT/MRI. Furthermore, microadenomas are not visualized on ${ }^{123} \mathrm{I}$-adosterol scintigraphy. On the other hand, a high number of nonfunctioning adrenal masses are found incidentally on CT/MRI. Unnecessary adrenalectomy, performed on the basis of CT images alone account for as many as $24.7 \%$ of cases [6].

It is challenging to detect the microadenomas less than $6 \mathrm{~mm}$ on CT or MRI, which account for nearly 50\% of APA [5]. It is also hard to evaluate whether the adenomas are functional or not, even if CT or MRI could detect them. Young et al. reported that $24.7 \%$ of 194 patients with adrenal adenomas, which underwent surgery due to preoperative CT findings, were non-functional [6]. In addition, microadenomas are not visualized on ${ }^{123}$ I-adosterol scintigraphy.

Adrenal venous sampling (AVS) is "gold standard" for evaluating the laterality, in which the concentration of aldosterone and cortisol were measured in blood samples from bilateral adrenal veins and inferior vena cava (IVC) through the catheters or microcatheters. It has been reported that the success rate of AVS is $94.3 \%$ [7], and the rate of major complications (e.g. rupture of adrenal veins, adrenal infarction, hematoma, adrenal vein thrombosis, and adrenal insuffi- 
ciency) is between $0.51 \%$ and $0.61 \%$ [8].

AVS blood sample analysis can be used to calculate plasma aldosterone concentration (PAC), lateralized ratio (LR) and contralateral ratio (CR). Each of these parameters has an established reference value for determining the laterality. Endocrinology physicians diagnosed the laterality of adrenal lesions based on these parameters comprehensively; however, it is often hard to assess it because there are many parameters. If it is known that which parameter is the most reliable in laterality assessment in AVS, more accurate diagnosis could be achieved and appropriate treatment could be performed. However, the best of our knowledge, there has been no reports determining the most accurate parameter in AVS.

Thus, the purpose of this study was to investigate the most accurate AVS parameter for determining the laterality in PA patients.

\section{Materials and Methods}

\subsection{Patients}

Written informed consent for the AVS procedure was obtained from all patients. The institutional review board of our university hospital approved this retrospective study, and the disclosure document can be found at the following URLhttp://www.ryukyu-radt.com/kanjya. Between April 2011 and December 2014, a total of 94 consecutive patients underwent AVS. All patients had been previously diagnosed with primary aldosteronism according to the Japan Endocrine Society guidelines 2009 [9]. At the primary screening, blood samples were obtained, and subsequently the plasma aldosterone concentration (PAC)/plasma renin activity (PRA) ratio (ARR) was calculated. If ARR was higher than 200, secondary screening was undertaken in which three medical load tests were performed (salineloading test, captopril-challenge test, and rapid ACTH stimulation test). If more than two of there tests were positive, the patient was diagnosed with PA. In order to obtain accurate AVS results, specific medications, including angiotensin converting enzyme inhibitors, angiotensin II receptor blockers, spironolactone, and eplerenone were stopped for at least 1 month prior to the procedure. $\mathrm{Pa}$ tients were continued on a calcium-channel blocker to control their blood pressure during the month prior to AVS.

Exclusion criteria included unsuccessful blood sampling $(n=16)$, lack of medical data $(n=6)$, and inadequate amount of sampling $(n=8)$. The remaining 64 patients (age: $50 \pm 12$ range $25-77,30$ males and 34 females) were enrolled in the study.

All patients underwent non-enhanced $(n=43)$ or enhanced $(n=21)$ abdominal computed tomography (CT) to demonstrate the anatomical location of the adrenal veins. The CT scanning parameters were as follows: slice thickness 5 $\mathrm{mm}$ or $1.25 \mathrm{~mm}$, peak $\mathrm{x}$-ray tube voltage $120 \mathrm{kVp}$, current $100-750 \mathrm{~mA}$ (automatic exposure control; AEC), field of view $170 \mathrm{~mm}$, beam width $0.625 \times 64=40$ mm (pitch: 1.375) in 64 detector-rows CT (LightSpeed VCT, GE Healthcare, 
Waukesha, Wisconsin, USA), and slice thickness $5 \mathrm{~mm}$ or $1 \mathrm{~mm}$, peak $\mathrm{x}$-ray tube voltage $120 \mathrm{kVp}$, current 100 - $570 \mathrm{~mA}$ (AEC), field of view $170 \mathrm{~mm}$, beam width $0.5 \times 160=80 \mathrm{~mm}$ (pitch: 0.875 ) in 320 detector-rows CT (Aquilion ONE, Toshiba Medical Systems, Tochigi, Japan).

\subsection{AVS Procedures}

All AVS procedures were performed in the angiography suite where cone-beam CT was available. The procedures were performed by 1 of 4 interventional radiologists (K.A. Y.I. T.K. and A.Y.) with extensive AVS experience (more than 30 cases each). Each operator performed AVS according to the following techniques and protocol.

A 5 or 6-Fr sheath was inserted through the left or right femoral vein under local anesthesia. A blood sample from the IVC was obtained below the confluence of the renal veins using the sheath or a catheter.

A 5.2-Fr reverse-curve catheter (Simons Shaped Carry; UTM, Aichi, Japan) was inserted into the left adrenal vein and venography was performed. In all patients, the left adrenal vein was found with the inferior phrenic vein. Blood samples were collected in the central adrenal vein (CAV) and the common trunk vein (CTV), which are above and below the inferior phrenic vein, respectively. When the blood sample was obtained from CAV, a microcatheter (2.7Fr Carry High-flow, UTM, Aichi, Japan or 2.8Fr Renegade Hi-Flo, Boston Scientific Corporation, Natick, MA, USA) was inserted through the orifice of the inferior phrenic vein (Figure 1 ).

A blood sample from the right adrenal vein (RAV) was also obtained. Cannulation of the right adrenal vein was difficult because of its small diameter, short length, acute angle of entry into the IVC, and anatomic variations. In general, a 5.2-Fr Mikaelsson catheter (Carry; UTM, Aichi, Japan) with one side hole was used, which provides easy blood sampling (Figure 2). When the cannulation was difficult, other catheter types were used (5Fr Cobra Shaped Carry or 4.2Fr RAV Carry; UTM, Aichi, Japan).

Blood samplings from IVC, CAV, CTV, and RAV were performed before and 30 minutes after intravenous administration of adrenocorticotropic hormone (ACTH; Cortrosyn ${ }^{\circledR}$ 0.25mg, Daiichi Sankyo Co., Ltd, Tokyo, Japan).

Criteria for adequate catheterization for AVS were as follows: 1) Adrenal venous cortisol concentration before ACTH stimulation $\geq 30 \mu \mathrm{g}$, after ACTH stimulation $\geq 200 \mu \mathrm{g}, 2$ ) Adrenal venous cortisol concentration before ACTH stimulation $>3$ times the cortisol concentration in blood from the IVC, 3) Adrenal venous cortisol concentration after ACTH stimulation $>5$ times the cortisol concentration in blood from the IVC.

No complications were observed in any of the 64 patients included in the study.

\subsection{Laterality Assessments}

Aldosterone and cortisol concentrations were measured in each site, and the 

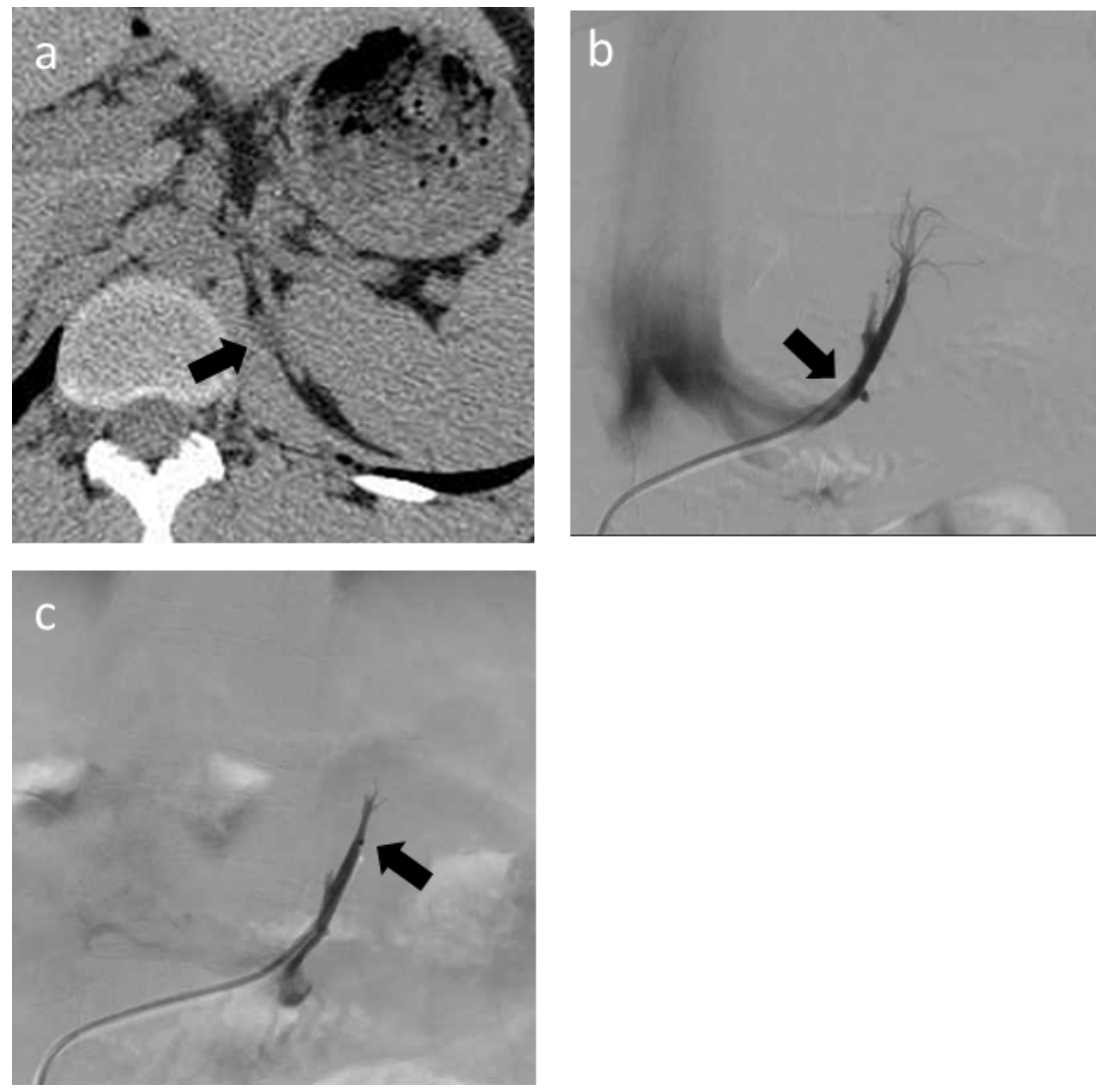

Figure 1. A 25-year-old man with primary aldsteronism (patient 54). Endocrinologists evaluated the parameters and determined that only left adrenal gland was involved. This patient underwent adrenalectomy, and histological analysis confirmed the adrenal adenoma. (a) Axial CT image with $1.25 \mathrm{~mm}$ slice thickness demonstrating a low-density nodule in the left adrenal gland (arrow). (b) Venogram of the common trunk of the left adrenal vein. A blood sample was obtained via a 5.2-Fr catheter in the common trunk vein (arrow). (c) A microcatheter was inserted through the confluence of the inferior phrenic vein, and a blood sample was obtained via the microcatheter (arrow).
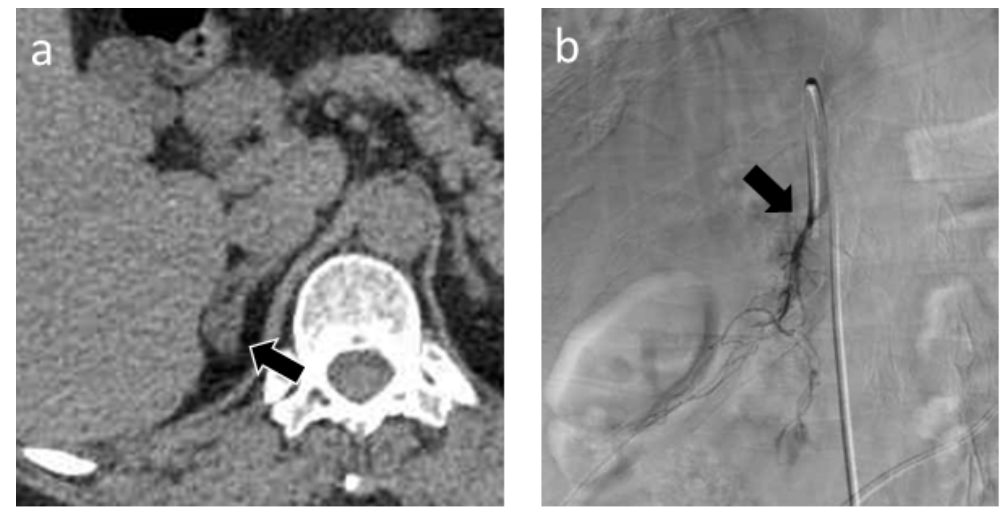

Figure 2. 58-year-old woman with primary aldsteronism (patient 43). Endocrinologists evaluated the parameters and determined that only right adrenal gland was involved. This patient underwent adrenalectomy, and histological analysis confirmed the adrenal adenoma. (a) Axial CT image with $1 \mathrm{~mm}$ slice thickness demonstrating a low-density nodule in the right adrenal grand (arrow). (b) Venogram of the right adrenal vein. A blood sample was obtained via a 5.2-Fr catheter in the right adrenal vein (arrow). 
following parameters were calculated: lateralized ratio [LR; aldosterone/cortisol ratio (ACR) on the high-value side/ACR on the low-value side], contralateral ratio (CR; ACR on the low-value side/ACR on the IVC ratio) and plasma aldosterone concentration (PAC).

The laterality was determined as follows: 1) If PAC was $>14,000 \mathrm{pg} / \mathrm{ml}$, the adrenal grand on this side was judged to be hypersecreting aldosterone, 2) If LR was $>2$, a unilateral lesion was present on the high-value side, 3) If CR was $<1$, a unilateral lesion was present on the high-value side (this indicates aldosterone production in the normal adrenal grand was suppressed). Based on these parameters, the final assessments of laterality was established by endocrinology physicians.

\subsection{Statistical Analysis}

The diagnostic accuracy of individual AVS parameters, including LR, CR, and PAC obtained from blood samples from the CTV/CAV/RAV during pre- and post-ACTH stimulation, was compared by receiver operating characteristic (ROC) analysis. The sensitivity, specificity, cut-off value, and $\mathrm{Az}$ value (the area under the ROC curve) were calculated. The statistical analyses were performed by Microsoft Excel 2007 (Microsoft, Tokyo, Japan) and the free statistical software "R" (R, v.2.6.1; The R Project for Statistical Computing; http://www.r-project.org/).

\section{Results}

Fifteen patients with unilateral lesion (25 - 69 years old (median, 53); six males, nine females; five were right-sided and 10 were left) (Table 1) and 49 patients with bilateral adrenal lesions were finally determined. All patients with unilateral lesion underwent adrenalectomy, and all lesions were histologically confirmed as adenomas $(n=12)$ or adrenal hyperplasias $(n=3)$. In 11 patients with unilateral lesion, it was possible to reduce or completely stop medications after the adrenalectomy. The other four were lost to follow up. Thirty-three of 49 patients with bilateral lesions were treated with medications (e.g. mineralocorticoid receptor antagonists) and improved clinically. The other 16 were lost to follow up.

The result of ROC analyses for all 64 patients are shown in Table 2. LR-CAV post-ACTH showed the highest detection rate for unilateral adrenal lesions (93.3\%; 14/15) with a sensitivity of 0.93 and a specificity of 0.84 , when the cutoff value was 2.5. One patient did not meet the LR-CAV post-ACTH criteria, but was diagnosed with a unilateral lesion by PAC post-ACTH.

The areas under the ROC curve (Az value) of LR-CTV post-ACTH (0.86), LRCAV post-ACTH (0.87), CR pre-ACTH (0.85), and CR post-ACTH (0.89) were higher than those obtained by other AVS methods (0.510 - 0.794). In particular, CR post-ACTH had the highest Az value, with a detection rate of $86.7 \%$ (13/14), a sensitivity of 0.98 and a specificity of 0.88 , when the cut-off value was set at 0.8 .

\section{Discussion}

Laterality of adrenal lesions in patients with PA can be determined using blood 
Table 1. Characteristics and adrenal venous sampling values of patients with unilateral adrenal lesions.

\begin{tabular}{|c|c|c|}
\hline \multicolumn{2}{|c|}{ No. of pt. } & 15 patients \\
\hline \multicolumn{2}{|c|}{ Age } & $25-69$ years old (median 53 ) \\
\hline \multicolumn{2}{|c|}{ Sex } & 6 males, 9 females \\
\hline \multicolumn{2}{|c|}{ Laterality } & 5 right-sided, 10 left-sided \\
\hline \multicolumn{2}{|c|}{ Diagnosis } & 12 adenomas, 3 hyperplasias \\
\hline \multirow{2}{*}{ Left-CAV-PAC } & Pre-ACTH & $283-20,000$ (mean 4614.3) \\
\hline & Post-ACTH & $1440-114,000($ mean 41,702$)$ \\
\hline \multirow{2}{*}{ Left-CTV-PAC } & Pre-ACTH & $152-5500$ (mean 2038.6) \\
\hline & Post-ACTH & 2120 - 99,100 (mean $24,319.3)$ \\
\hline \multirow{2}{*}{ Right-PAC } & Pre-ACTH & $86-81,000$ (mean 8738.6$)$ \\
\hline & Post-ACTH & $290-445,000($ mean 50,043.3) \\
\hline \multirow{2}{*}{ LR-CAV } & Pre-ACTH & $0.5-56.7($ mean 17.8$)$ \\
\hline & Post-ACTH & $0.2-84($ mean 13.7$)$ \\
\hline \multirow{2}{*}{ LR-CTV } & Pre-ACTH & $0.2-67.2($ mean 15.2$)$ \\
\hline & Post-ACTH & $0.2-92.7$ (mean 14.8) \\
\hline \multirow{2}{*}{ CR } & Pre-ACTH & $0.2-6.9($ mean 1.3$)$ \\
\hline & Post-ACTH & $0.1-14.1($ mean 1.3$)$ \\
\hline
\end{tabular}

Abbreviations: ACTH, adrenocorticotropic hormone; CAV, central adrenal vein; PAC, plasma aldosterone concentration; CTV common trunk vein; LR, lateralized ratio; CR, contralateral ratio.

Table 2. Receiver operating characteristic (ROC) analysis of values obtained during adrenal venous sampling.

\begin{tabular}{ccccccc}
\hline Evaluation methods & DR & $(\%)$ & Az value & Sens & Spec & Cut-off \\
\hline left-CAV-PAC pre-ACTH & $1 / 15$ & $(7)$ & 0.597 & 0.667 & 0.551 & 1490 \\
left-CAV-PAC post-ACTH & $10 / 15$ & $(67)$ & 0.518 & 0.6 & 0.592 & 28300 \\
left-CTV-PAC pre-ACTH & $0 / 15$ & $(0)$ & 0.589 & 0.667 & 0.571 & 971 \\
left-CTV-PAC post-ACTH & $9 / 15$ & $(60)$ & 0.513 & 0.959 & 0.4 & 5300 \\
right-PAC pre-ACTH & $3 / 15$ & $(20)$ & 0.646 & 0.612 & 0.733 & 1190 \\
right-PAC post-ACTH & $6 / 15$ & $(40)$ & 0.708 & 0.877 & 0.666 & 14600 \\
LR-CAV pre-ACTH & $12 / 15$ & $(80)$ & 0.787 & 0.666 & 0.776 & 4.1 \\
LR-CAV post-ACTH & $14 / 15$ & $(93)$ & 0.865 & 0.933 & 0.836 & 2.5 \\
LR-CTV pre-ACTH & $12 / 15$ & $(80)$ & 0.794 & 0.8 & 0.653 & 2 \\
LR-CTV post-ACTH & $13 / 15$ & $(87)$ & 0.86 & 0.866 & 0.816 & 2.2 \\
CR pre-ACTH & $10 / 15$ & $(67)$ & 0.851 & 0.816 & 0.733 & 1.3 \\
CR post-ACTH & $13 / 15$ & $(87)$ & 0.889 & 0.98 & 0.867 & 0.8 \\
\hline
\end{tabular}

Abbreviations: DR, Detection rate; Sens, sensitivity; Spec, specificity; Cut-off, cut-off value; CAV, central adrenal vein; PAC, plasma aldosterone concentration; ACTH, adrenocorticotropic hormone; CTV, common trunk vein; $L R$, lateralized ratio; $C R$, contralateral ratio. 
samples obtained from AVS. Various AVS evaluation methods have established reference values for determining laterality. However, there is no consensus regarding how these evaluation methods should be compared. It is important to establish a definite set of criteria for the assessment of AVS methods in patients with PA, as laterality determines PA management.

In the present study, LR-CAV post-ACTH yielded the highest detection rate of unilateral lesions $(93.0 \%)$ with higher sensitivity and specificity than other values. CR post-ACTH had the highest Az value, with a detection rate of $86.7 \%$, a sensitivity of 0.980 , and a specificity of 0.889 , when the cut-off value was set at 0.8 . These results were superior to those of LR-CAV post-ACTH (sensitivity, 0.933; specificity, 0.836), although the detection rate of LR-CAV post-ACTH was superior to that of CR post-ACTH. These findings suggest that both LR-CAV post-ACTH and CR post-ACTH can be used to determine the laterality of PA.

It has been difficult to come to a consensus regarding the proper position of the catheter tip for blood sampling from the left adrenal vein. According to the Japanese guidelines [9], the catheter tip should be placed distal to the orifice of the inferior phrenic vein. However, worldwide consensus has not yet been established. Therefore, in some institutions, left adrenal blood samples are obtained at two locations (CTV and CAV), similar to our procedure. A microcatheter is often required for collection of these samples. We found that LR-CAV post-ACTH had the highest detection rate of unilateral disease. Blood samples from the CAV had a higher detection rate than samples from the CTV. In a previous study [10], the ACR was not significantly different between the CAV and CTV, although higher levels of cortisol and aldosterone were measured in upstream samples. Our study included a larger patient population, which may account for the different findings.

Our results suggest that an integrated evaluation of LR-CAV post-ACTH and $\mathrm{CR}$ post-ACTH may be the most accurate way to diagnose unilateral disease. Although the use of ACTH stimulation has been controversial, we found that higher $\mathrm{Az}$ values were obtained after ACTH stimulation. We therefore suggest that blood sampling after ACTH stimulation should be performed, despite the increase in cost and AVS examination time.

Our study had some limitations. First, we did not obtain pathological confirmation of all adrenal lesions because patients diagnosed with bilateral disease did not undergo surgical resection. However, follow-up evaluation and treatment response confirmed the diagnosis. Second, the sample size was relatively small, and a larger study is needed to confirm our results. Third, our study included selection bias, as only those patients who were surgical candidates were selected for inclusion.

\section{Conclusion}

In conclusion, CR post-ACTH and LR-CAV post-ACTH by AVS provide accurate evaluation and high detection rates in PA patients. It is possible to give more accurate diagnosis and more appropriate management for PA patients by 
evaluating these reliable parameters mainly in laterality assessment, which leads to high-quality prognosis.

\section{References}

[1] Funder, J.W., Carey, R.M., Fardella, C., Gomez-Sanchez, C.E., Mantero, F., Stowasser, M., Young Jr., W.F. and Montori, V.M. (2008) Case Detection, Diagnosis, and Treatment of Patients with Primary Aldosteronism: An Endocrine Society Clinical Practice Guideline. The Journal of Clinical Endocrinology \& Metabolism, 93, 3266-3281. https://doi.org/10.1210/jc.2008-0104

[2] Nishikawa, T., Saito, J. and Omura, M. (2007) Prevalence of Primary Aldosteronism: Should We Screen for Primary Aldosteronism before Treating Hypertensive Patients with Medication? Endocrine Journal, 54, 487-495.

[3] Milliez, P., Girerd, X., Plouin, P.F., Blacher, J., Safar, M.E. and Mourad, J.J. (2005) Evidence for an Increased Rate of Cardiovascular Events in Patients with Primary Aldosteronism. Journal of the American College of Cardiology, 45, 1243-1248. https://doi.org/10.1016/j.jacc.2005.01.015

[4] Daunt, N. (2005) Adrenal Vein Sampling: How to Make It Quick, Easy, and Successful. Radiographics, 25, 143-158. https://doi.org/10.1148/rg.25si055514

[5] Omura, M., Saito, J., Yamaguchi, K., Kakuta, Y. and Nishikawa, T. (2004) Prospective Study on the Prevalence of Secondary Hypertension among Hypertensive Patients Visiting a General Outpatient Clinic in Japan. Hypertension Research, 27, 193-202. https://doi.org/10.1291/hypres.27.193

[6] Young, W.F., Stanson, A.W., Thompson, G.B., Grant, C.S., Farley, D.R. and Van Heerden, J.A. (2004) Role for Adrenal Venous Sampling in Primary Aldosteronism. Surgery, 136, 1227-1235. https://doi.org/10.1016/j.surg.2004.06.051

[7] Kądziela, J., Prejbisz, A., Michałowska, I., Kołodziejczyk-Kruk, S., Schultze Kool, L., Kabat, M., Janaszek-Sitkowska, H., Toutounchi, S., Gałązka, Z., Ambroziak, U., Bednarczuk, T., Ptasińska-Wnuk, D., Hoffmann, M., Januszewicz, M., Januszewicz, A. and Witkowski, A. (2017) A Single-Centre Experience of the Implementation of Adrenal Vein Sampling Procedure: The Impact on the Diagnostic Work-Up in Primary Aldosteronism. Kardiologia Polska, 75, 28-34.

https://doi.org/10.5603/KP.a2016.0166

[8] Rossi, G.P., Barisa, M., Allolio, B., Auchus, R.J., Amar, L., Cohen, D., Degenhart, C., Deinum, J., Fischer, E., Gordon, R., Kickuth, R., Kline, G., Lacroix, A., Magill, S., Miotto, D., Naruse, M., Nishikawa, T., Omura, M., Pimenta, E., Plouin, P.F., Quinkler, M., Reincke, M., Rossi, E., Rump, L.C., Satoh, F., Schultze Kool, L., Seccia, T.M., Stowasser, M., Tanabe, A., Trerotola, S., Vonend, O., Widimsky Jr., J., Wu, K.D., Wu, V.C. and Pessina, A.C. (2012) The Adrenal Vein Sampling International Study (AVIS) for Identifying the Major Subtypes of Primary Aldosteronism. The Journal of Clinical Endocrinology \& Metabolism, 97, 1606-1614. https://doi.org/10.1210/jc.2011-2830

[9] Nishikawa, T., Omura, M., Satoh, F., Shibata, H., Takahashi, K., Tamura, N. and Tanabe, A. (2011) Guidelines for the Diagnosis and Treatment of Primary Aldosteronism-The Japan Endocrine Society 2009. Endocrine Journal, 58, 711-721. https://doi.org/10.1507/endocri.EJ11-0133

[10] Takada, A., Suzuki, K., Mori, Y., Hyodo, R., Kawakami, K., Okochi, Y. and Naganawa, S. (2013) Comparison of the Central Adrenal Vein and the Common Trunk of the Left Adrenal Vein for Adrenal Venous Sampling. Journal of Vascular and Interventional Radiology, 24, 550-557. https://doi.org/10.1016/j.jvir.2013.01.007 